Ann. Génét. Sél. anim., I975, 7 (2), I39-144.

\title{
A 589
}

\section{VARIATION INTERRACIALE DE LA TAILLE DU CHROMOSOME Y CHEZ BOS TAURUS L.}

\author{
E. P. CRIBIU \\ avec la collaboration technique de Jeannine Boscher \\ U. N.C.E. I. A. et Laboratoire de Génétique factorielle, \\ Centre national de Recherches zootechniques, I. N. R. A., \\ 78350 Jouy en Josas
}

\section{RÉSUMÉ}

Une étude portant sur la longueur du chromosome $Y$ a été entreprise chez 87 taureaux appartenant à cinq races françaises : Normande, F.F.P.N., Charolaise, Montbéliarde et Maine-Anjou. La longueur relative de l'Y a été calculée par rapport à la somme de la première paire autosomale et de $1^{\prime} X$, seuls chromosomes aisément identifiables dans le caryotype bovin.

Il apparaît des différences interraciales significatives de la taille de l'Y pour les cinq races étudiées : le plus grand étant chez les Charolais et les Montbéliards et le plus petit chez les Normands et les Maine-Anjou.

\section{INTRODUCTION}

Les anomalies du chromosome $\mathrm{X}$ sont associées chez Bos taurus $\mathrm{L}$. et chez la plupart des Mammifères à des syndromes cliniques variés et des troubles de la fertilité (Bouters et al., I974; ScotT et GrEGory, I965; RIECK et al., I970). Bien que très peu d'études aient été faites sur le chromosome $Y$ des bovins, celui-ci ne semble pas, quand il est surnuméraire (DoBRIYANOv et KonstamTrNov, I970) ou anormalement long (FECHHEIMER, I973 et CRIBIU et PoPESCU, I974) entraîner des malformations physiques.

$\mathrm{Du}$ fait de la présence sur le $\mathrm{X}$ de gènes autosomaux et de déterminants sexuels, il n'est pas surprenant que les anomalies de ce chromosome soient plus graves en 
terme de fertilité et de malformations physiques ou mentales, que celles du chromosome Y qui, lui, ne contient pas ou peu de gènes autosomaux (OHNo et al., I964).

Il semble donc logique que, si un polymorphisme chromosomique devait être toléré par une espèce, il apparaîtrait plutôt sur l'Y, qui est le moins génétiquement actif et dont la perte n'est pas, d'habitude, léthale.

L'existence d'un polymorphisme pour la taille du chromosome Y a été mis en évidence chez plusieurs espèces : le Hamster Syrien (LEHMAN et al., I963), le Rat (Hungerford et Noweli,, I963), la Souris (MATThey, I963 et I964 ; ChI et WEIR, I974) et 1'Homme (CoHEN et al., I966; UNNERUs et al., I967 et DE LA TORRE et GimenEZ-Martin, I970). Zhivalev et Goldpman (I973) font mention de l'existence d'un polymorphisme du chromosome $\mathbf{Y}$ dans une population bovine en Russie.

Une étude biométrique sur ce chromosome, dans plusieurs races bovines, fait l'objet de cette note.

\section{MATÍRIELS ETT MÉTHODES}

Notre étude a porté sur 87 taureaux de cinq races françaises : Maine-Anjou (8), Normande (25) F.F.P.N. (20), Charolaise (16) et Monbéliarde (18). Ces animaux, phénotypiquement normaux et ne présentant pas de troubles de fertilité, provenaient de divers centres d'insémination artificielle.

Après avoir effectué des cultures de sang entier, selon la technique de DE Grouchy et ai. (1964), les meilleurs métaphases, dont les critères de sélection étaient : une bonne dispersion, l'absence de chevauchement et un degré moyen de contraction, ont été photographiées sur film Kodak microfile avec une caméra Leitz-Ortholux.

On a mesuré la plupart des photographies sans connaître a priori la race de l'animal dont elles étaient issues.

Sur chaque photographie nous avons identifié les chromosomes qui devaient nous servir de référence : la paire no ${ }^{\circ}$ parce qu'elle est composée par les deux plus grands des autosomes du caryotype bovin (CRIBIU et PopesCU, r974), et les deux chromosomes X et Y facilement repérables en raison de leur morphologie.

Les mesures de ces quatre chromosomes ont été faites à l'aide d'un compas à pointe sèche et d'un pied à coulisse au $\mathrm{I} / 5^{\circ} \mathrm{e}$ directement sur les photographies selon une méthode décrite antérieurement (CRIBIU, 1974).

Nous avons mesuré deux à trois cellules par animal (soit 204 métaphases au total), les critères de qualité énoncés précédemment nous ayant fait éliminer un très grand nombre de photographies.

Pour chaque cellule nous avons calculé la longueur relative $\left(\mathrm{Y}_{c}\right)$ et l'indice centromérique (IC) du chromosome $\mathrm{Y}$ selon les relations suivantes:

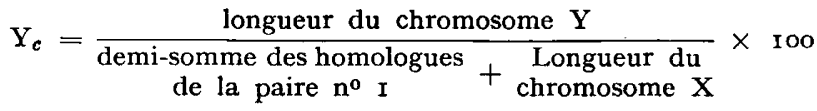

$$
\begin{aligned}
& \mathrm{IC}=\frac{\text { longueur du bras court }}{\text { longueur totale }} \times \text { roo. }
\end{aligned}
$$

Afin de vérifier l'usage de $Y_{c}$ comme longueur représentative du chromosome $Y$ nous avons pris, parmi les 204 cellules étudiées, 99 métaphases pour lesquelles nous avons mesuré les 60 chromosomes et exprimé la longueur relative $\left(\mathrm{Y}_{r}\right)$ selon la formule :

$$
\mathrm{Y}_{r}=\frac{\text { longueur du chromosome } \mathrm{Y}}{\underset{5^{8} \text { autosomes }}{\text { demi-somme des }}+\text { longueur du chromosome } \mathrm{X}} \times \text { I ooo. }
$$

Ainsi pour 99 métaphases nous avons eu deux valeurs $\left(\mathrm{Y}_{c}\right.$ et $\left.\mathrm{Y}_{r}\right)$ exprimant la longueur du_chromosome $\mathrm{Y}$ pour une même cellule. 
Pour vérifier la représentativité de $\mathrm{Y}_{c}$ comme longueur relative du chromosome $\mathrm{Y}$, nous avons calculé pour les 99 cellules la corrélation entre $Y_{c}$ et $Y_{r}$ : celle-ci étant positive et hautement significative $(r=+0,96 \mathrm{P}<0,00 \mathrm{r})$, nous avons utilisé comme longueur relative du chromosome $\mathrm{Y}$ la variable $Y_{c}$ pour l'ensemble des cellules étudiées.

Nous avons calculé, à partir de la longueur relative et de l'indice centromérique du chromosome Y de chacune des 204 cellules, les valeurs moyennes par taureau puis, à l'aide de ces dernières, les moyennes par race.

Nous avons effectué pour la longueur du chromosome $\mathrm{Y}$ une analyse de variance selon un modèle hiérarchique à 2 niveaux (DARRE et al., 1974) afin de tester d'une part la signification des différences entre taureaux d'une même race et d'autre part la signification des différences entre races.

Un test de Tukey a enfin été employé pour séparer les moyennes des longueurs relatives des chromosomes $\mathrm{Y}$ de chaque race.

\section{RÉSULTATS}

Dans le tableau I sont données les moyennes, variances et coefficients de variation de la longueur relative et de l'indice centromérique du chromosome $\mathrm{Y}$ dans les cinq races.

\section{TABLEAU I}

Moyenne, variance et coefficient de variation de la longueur relative et de l'indice centromérique du chromosome $Y$ dans les cinq races

\begin{tabular}{|c|c|c|c|c|c|c|c|}
\hline \multirow{2}{*}{ Races } & \multirow{2}{*}{$\begin{array}{l}\text { Nombre } \\
\text { de cellules }\end{array}$} & \multicolumn{3}{|c|}{ Longueur du chromosome $\mathrm{Y}$} & \multicolumn{3}{|c|}{$\begin{array}{l}\text { Indice centromérique } \\
\text { du chromosome } \mathrm{Y}\end{array}$} \\
\hline & & $\overline{\mathbf{Y}}$ & Variance & CV $\left({ }^{1}\right)$ & IC & Variance & $\mathrm{CV}(\mathbf{1})$ \\
\hline Maine-Anjou (8) & 25 & 20,18 & 1,31 & 5,64 & 42,83 & 6,03 & 5,72 \\
\hline Normande (25) & 53 & 20,61 & 6,38 & 12,20 & 42,35 & 8,03 & 6,68 \\
\hline F.F.P.N. (20) & 47 & $21,0 \%$ & 4,99 & 10,50 & 44,54 & 7,67 & 6,19 \\
\hline Charolaise (16) & 43 & 23,02 & 6,67 & 11,2 & 41,54 & 13,42 & 8,81 \\
\hline Montbëliarde (18) & 36 & 23,70 & 3,61 & 8,00 & 42,07 & 14,27 & 8,96 \\
\hline
\end{tabular}

(1) $\mathrm{CV}$ : Coefficient de variation $=\frac{\text { écart-type }}{\text { moyenne }} \times 100$.

Le tableau 2 représente l'analyse de variance effectuée pour la longueur du chromosome Y. La même analyse employée avec l'indice centromérique n'a pas donné de $\mathrm{F}$ significatifs au seuil de $5 \mathrm{p}$. Ioo que ce soit à l'intérieur ou entre les races.

En utilisant le test de Tukey il apparaît deux groupes : Mainz-Anjou, Normand et F.F.P.N. d'une part et Montbéliard et Chxrolais d'autre part.

Les différences de longueurs des chromosomes $\mathrm{Y}$ des différentes races sont illustrées par la figure I. 


$$
\begin{aligned}
& \operatorname{lnx} x^{n n x_{x}} \\
& \ln x_{x} \\
& \operatorname{nax} \times \operatorname{nos} x
\end{aligned}
$$




\section{DISCUSSION}

L,es différentes analyses statistiques suggèrent que, s'il existe une variation interraciale pour la longueur du chromosome $\mathrm{Y}$, par contre, il ne semble pas y avoir de variation intra-raciale. Les Maine-Anjou et les Normands auraient un Y plus petit que les Charolais et les Montbéliards, la taille de celui des F.F.P.N. étant intermédiaire. JORGE (r974) trouva des différences semblables entra les Frisons et les Charolais de même que HANSEN et ELLEBBy (r975) entre les Rouges Danois et les Pies noires Danois d'une part et les Charolais de l'autre.

En général, les variations de longueur d'un chromosome sont le reflet de différences de quantité et de masse de matériel génétique impliquant des remaniements structuraux tels que les duplications, délétions ou translocations, par exemple. L'absence de différence significative des indices centromériques entre les races indique qu'il y a eu variation de longueur sur les deux bras du $\mathrm{Y}$.

Cependant l'observation du chromosome $\mathbf{Y}$ d'animaux appartenant à ces cinq races avec les techniques de "bandes " $G$ (Popescu, communication personnelle) ne montre pas de différences quant au nombre et à la structure des bandes.

Ceci nous amène à envisager 1'hypothèse suivant laquelle cette variation interraciale de l'Y serait due à un degré différent de spiralisation de la chromatine (WENNSTRÖM et DE LA CHAPELLE, I963). Il n'y aurait pas alors de changement de quantité de matériel génétique et, par la même, pas de modification de l'indice centromérique.

Le test de Tukey a mis en évidence l'existence de deux groupes comprenant l'un les Maine-Anjou, Normands, F.F.P.N. et l'autre les Charolais et Montbéliards. Ces résultats ne sont pas en désaccord avec ceux de GroscLAUDE (I975) concernant les groupes sanguins et la parenté existant entre Charolais et Montbéliards. De plus, si l'on se réfère à la fréquence de la fusion centrique $\mathbf{I} / 29$ dans les différentes races françaises (POPESCU et BoschER, r974), on constate que celle-ci est nulle parmi les races du premier groupe alors qu'elle a un taux relativement élevé chez les Montbéliards (POPESCU, I97I) et les Charolais (HARVEY, I974).

Reçu pour publication en avril 1975.

\section{REMERCIEMENTS}

Je tiens à remercier MM. P. Mérat, C. P. Popescu (Laboratoire de Génétique factorielle, C. N. R. Z., 78350 Jouy en Josas) et D. L. Pollock (The Edinburgh School Agriculture) pour leurs suggestions et leurs critiques sur l'analyse et la rédaction de ce travail.

\section{SUMMARY}

BREED DIFFERENCES IN THE LENGTH OF THE BOVINE Y CHROMOSOME

A study on the length of the $\mathrm{Y}$ chromosome in 87 bulls from five French breeds : Norman, F.F.P.N., Charolais, Montbéliard and Maine-Anjou was made. The $\mathrm{Y}$ chromosome, the $\mathrm{X}$ and the largest autosomal pair were mesured. 
Analysis of the data demonstrated that the mean length of the $\mathrm{Y}$ chromosome in the Charolais and Montbeliard bulls was significantly greater than that of the other breeds.

\section{RÉFÉRENCES BIBLIOGRAPHIQUES}

Bouters R., Bonte P., Spincemaille J., Vandeplassche M., 1974. The chromosomes of domestic animals. II. Chromosomal abnormalities associated with reproductive disorders. Vlaams diergeneesk. Tijdschr., 43, 85-91.

CHI N. Y., WeIr J. A., r974. Autosomal control over length of the Y-chromosome in mice. Genetics, 77, n⿳ I/Part 2, Supplément S. Ir.

Cohen M. M., Shaw M. W., Maccluer J. W., I966. Racial differences in the length of the human $Y$ chromosomes. Cytogenetics, 5, 34-52.

CRIBIU E. P., I974. Observations sur les chromosomes somatiques chez Bos taurus $L$. Thèse de doctorat de $3^{\mathrm{e}}$ cycle, Université de Paris VI.

Cribiu E. P., Popescu C. P., r974. L'Idiogramme de Bos taurus L. Ann. Génét. Sél. anim., 6, 291-296.

Cribiu E. P., Popescu C. P., I 974. Un cas de chromosome Y anormalement long chez Bos taurus L. Ann. Gênét. Sél. anim., 6, 387-390.

Darré R., Muorwell M., Berland H. M., Queinnec G., 1974. Variation de la longueur relative du chromosome Y chez les bovins. I er Congr. mond. génét. appl. Élev., Madrid, 3, I25-1 30.

De Grouchy J., Roubin M., Passage E., I964. Microtechnique pour l'étude des chromosones humains à partir d'une culture de leucocytes sanguins. Ann. Génét., 7, 45.

De La Torre C., Gimenez-Martin G., I97o. The human Y chromosome in the Spanish population. J. Genet. hum., 18, 235-254.

Dobriyanov D., Konstamtinov G., I97o. A case of mosaicism of the 58 AXY, 58 AXYY type in a male calf of the Bulgarian Brown cattle breed. C. R. Acad. Sci. agric. Bulg., 3, $27 \mathrm{I}-276$.

Fесннеimer N. S., r973. A cytogenetic survey of young bulls in the U. S. A. Vet. Rec., 93, 535-536.

G rosclaude F. Laboratoire de Génétique biochimique. Département de Génétique animale, C. N. R. Z., 78350 Jouy en Josas, 1975, communication personnelle.

Hansen K. M., Elleby F., 1975. Chromosome investigation of danish A. I. beef bulls. Nord. Vet. Med., 27, 102-106.

Harvey J. A., I974. Chromosome analysis of cattle populations. Vet. Rec., 94, 227.

Hungerford D. A., Nowell P. C., I963. Sex chromosome polymorphism and the normal caryotype in three strains of the Laboratory rat. $J$. morph., 113, 275-285

JORGE W., I974. Chromosome study of some breeds of cattle. Caryologia, 27, 325-329.

Lehman J. M., Macpherson I., Moorhead P. S., 1963. Karyotype of the Syrian hamster. J. nat. Cancer Inst., 31, 639-650.

Matthey R., 1963. Cytologie comparée et polymor phisme chromosomique chez les $M$ us africains appartenant aux groupes Bufotriton et Minutoides (Mammalia Rodentia). Cytogenetics, 2, 290-322.

Matthey R., r964. Évolution chromosomique et spéciation chez les Mus du sous-genre Leggada, Gray 1837. Experientia, 20, 657-665.

Ohno S., BecaK W., BecaK H. L., I964. X-autosome ratio and the behavior pattern of individual $\mathrm{X}$-chromosome in placental mammals. Chromosoma, 15, $\mathrm{x}-30$.

Popescu C. P., r97r. Deux cas nouveaux de fusion centrique chez les bovins. Ann. Génét. Sél. anim., 3, $521-525$.

Popescu C. P., Boscher J, 1974. Observations sur une fusion centrique chez les bovins (Bos taurus L). Ier Congr. mond. génét. appl. Élev., Madrid, 3, I65-I68.

Rieck G. W., Hohn H., Herzog A., r97o. X-Trisomie beim Rind mit Anzeichen familiarer Disposition für Meiosestörungen. Cytogenetics, 9, 40r-409.

Scotr C. D., Gregory P. W., 1965. An XXY trisomic in an intersex of Bos taurus. Genctica, 52, 473-474.

Unnerus V., Fellman J., De La Chapelle A., r967. The length of the human Y chromosome. Cytogenetics, 6, 213-227.

Wennstróm J., De la Chapelle A., r963. Elongation as the possible mechanism of origin of large human Y chromosomes. Hereditas, 50, 345-350.

Zhivalev I. K., Goldman I. L., I973. Polymorphisme du chromosome Y dans une population de bovins. Dokl. Vsesojuz. Akad. Sel' skokhoz. Nauk. V. I., Lenina, U. R. S. S., 2, 30-32. 